\title{
Dislocation arrangement in small crystal volumes determines power-law size dependence of yield strength
}

\author{
R. Gu and A.H.W. Ngan ${ }^{\S}$ \\ Department of Mechanical Engineering, The University of Hong Kong, \\ Pokfulam Road, Hong Kong, P.R. China \\ § Corresponding author (email: hwngan@hku.hk)
}

\begin{abstract}
It is by now well-known that micron-sized metallic crystals exhibit a smaller-beingstronger size effect: the yield strength $\sigma$ varies with specimen size $D$ approximately as a power law $\sigma \sim D^{-m}$, and the exponent $m$ has been found to vary within a range of $\sim 0.3$ to $\sim 1.0$ for different metals. However, little is known about why such a power law comes into play, and what determines the actual value of the exponent $m$ involved. This work shows that if the yield strength is determined by the Taylor interaction mechanism within the initial dislocation network, then for the size dependence of strength to be of the power-law relation observed, it is necessary for the mesh lengths $L$ of the dislocation network to be power-law distributed, i.e. $p(L) \sim L^{-q}$. In such a case, the exponent $m$ of the size effect is predicted to be inversely proportional to the sum of $q$ the exponent of the mesh-length distribution and $n$ the exponent of the dislocation velocity vs stress law. To verify these predictions, compression experiments on aluminum micro-pillars with different pre-strains from 0 to $15 \%$ were carried out. The different pre-strains led to different initial dislocation networks, as well as different exponent $m$ in the size dependence of strength. Box-counting analyses of transmission electron micrographs of the initial dislocation networks showed that the 2-D projected dislocation patterns were approximate fractals. On increasing pre-strain, the exponent $m$ for the size dependence of strength was found to decrease while the fractal dimension of the initial dislocation patterns increased, thus verifying the inverse relationship between the two quantities. These findings show that the commonly observed power-law scaling of strength with size is due to an approximate power-law distribution of the initial dislocation mesh lengths, which also appears to be a robust feature in deformed metals.
\end{abstract}


Furthermore, for a given metal, it is the exponent $q$ of the initial mesh-length distribution which determines the value of the exponent $m$ in the size dependence of strength.

Keywords: Yield strength; dislocations; size effect; nanoindentation; transmission electron microscopy 


\section{Introduction}

That smaller metallic specimens are stronger was first reported as early as in the 1950's in tensile tests of whiskers (Brenner, 1956a, b, 1957) but the modern-day interest on this subject was re-triggered by Uchic et al.'s compression experiments on micron-scale metallic pillars using a state-of-the-art combination of focused-ion-beam machining and nanoindentation tools (Uchic et al., 2004). The large volume of literature (Dimiduk et al., 2005; Greer et al. 2005; Dimiduk et al., 2007; Frick et al., 2008; Greer and De Hosson, 2011; Han et al., 2010; Kim and Greer, 2009; Kim et al., 2010; Lee et al., 2009; Ng and Ngan, 2008b; Sun et al., 2011; Volkert and Lilleodden, 2006; Ye et al., 2011; Kunz et al. 2011) so far has indicated that the yield strength $\sigma$ of micron-sized metals scales with size $D$ according to a power law:

$$
\sigma \sim D^{-m}
$$

By surveying a number of studies on face-centered cubic (fcc) metals, Dou and Derby (Dou and Derby, 2009) also proposed a universal form of equation (1): $\sigma / \mu=A(D / b)^{-m}$, where $\mu$ is the shear modulus, $b$ the magnitude of Burgers vector, and $A$ a constant. In the DouDerby relation, and the mean value of $m$, among the metals surveyed, was $\sim 0.66$, but scatter about such a mean value was large. Greer and De Hosson (Greer and De Hosson, 2011) surveyed additional reports and concluded that $m$ varies from 0.6 to 1 for different fcc metals. In fact, if body-centered cubic and hexagonal close-packed metals are included, the range of $m$ is further widened; for example, $m$ was found to be $~ 0.43$ for Ta (Kim et al., 2010), 0.44 for Mo and W(Kim et al., 2010), 0.5 for Ti (Sun et al., 2011) and 0.64 for Mg (Ye et al., 2011) micro-pillars, and $\sim 1$ for Fe whiskers (Brenner, 1957). The known range of $m$ is therefore from $\sim 0.3$ to $\sim 1.0$. For the same metal, different studies also reported different values of $m$. For example for Au $m$ was reported to be 0.61 by Volkert et al. (Volkert and Lilleodden, 2006) and 0.41 by Lee et al.(Lee et al., 2009); for Ni $m$ was reported to be 0.64 by Dimiduk et al. (Dimiduk et al., 2005) and 0.69 by Frick et al. (Frick et al., 2008); for $\mathrm{Al} m$ was reported to be 0.92 (Ng and Ngan, 2008) and 0.63 by Kunz et al. (Kunz et al. 2011).

Several groups have proposed models to explain the smaller-being-stronger size effect of strength in the micro-regime. Leaving aside further strengthening that may arise due to the presence of strain gradients in the deformation geometry (Huang et al., 2000a; Huang et al., 
2000b; Nix and Gao, 1998), two groups of models apply to strain-gradient-free situations as in uniaxial tests, namely, dislocation starvation (Greer and Nix, 2006; Greer et al., 2005) and dislocation source models (El-Awady et al., 2009; Norfleet et al., 2008; Parthasarathy et al., 2007; Rao et al., 2008; Rao et al., 2007). In the "starvation” model (Greer and Nix, 2006; Greer et al., 2005), dislocations zip through the small crystal volume without producing accumulation or multiplication, and so the crystal is kept in a dislocation-starved state, with strength dominated by dislocation nucleation at free surfaces or homogeneously. A direct proof of the sustained dislocation starved state during deformation has been provided by Shan et al.'s in situ experiment on Ni nano-pillars (Shan et al., 2008). A spatio-temporal stretching model using molecular-dynamics computed nucleation rates also revealed a weak but definite smaller-beingstronger size effect (Zuo and Ngan, 2006), which has recently gained some experimental support from compression tests on $\mathrm{Ni}_{3} \mathrm{Al}$ nano-cubes (Maaß et al., 2012).

However, the dislocation starved state is unlikely to arise in specimens containing a high amount of existing dislocations, where a traversing dislocation becomes more likely to interact with some of the existing dislocation segments to produce strengthening and dislocation multiplication. Relevant to this latter regime where the starved state is not achieved is a group of models focusing on the operation and propensity of dislocation sources in small confined volumes. The "source truncation" model (Parthasarathy et al., 2007; Rao et al., 2007) assumes that the sample size determines the truncated dislocation source lengths, so that the source operational strength becomes higher as size decreases. Dislocation dynamics simulations using presumed initial dislocation distributions (El-Awady et al., 2009; Parthasarathy et al., 2007) have also predicted a size effect on strength. An analytical model using a Boltzmann factor form for the dislocation source production rate (Ng and Ngan, 2008b) also predicted a size effect of the form $\sigma=A-B \ln D$, where $A$ and $B$ are constants. However, all these models do not explicitly account for the power-law scaling of strength as in equation (1).

The purpose of this paper is to show that the universal power-law scaling of strength with size is a consequence of a power-law distribution of the mesh lengths in the initial dislocation network under the non-starved state. The theoretical foundation is first discussed in the next section, followed by experimental verification based on micro-pillar compression experiments in aluminum. 


\section{Theoretical Considerations}

The wide applicability of equation (1) to different materials suggests that the underlying mechanism is equally widely applicable. Recently, one of us proposed a model, based on Taylor interactions within the existing dislocation network, to show that if the mesh-length distribution in the initial dislocation network is a power-law, then the yield strength will vary with size as a power law (Ngan, 2011). Here, we show that the reverse is also true, i.e. if the yield strength varies with size as a power law, then the mesh-length distribution should also be a power law. Also, we further develop the model here to enable comparison with experimental observations, which are discussed in the next section.

\subsection{Ensemble sampling of Taylor interactions in a 3-D dislocation network}

To capture the stochastic nature of deformation of micro-crystals, let us consider an ensemble of a large number of macroscopically identical experiments. A survival probability $F(\sigma)$ can be defined from this ensemble as the fraction of samples that remain un-yielded at a current applied stress level $\sigma(\mathrm{Ng}$ and Ngan, 2008a, b). $F(\sigma)$ is a monotonically decreasing function because as $\sigma$ increases the samples will yield one after another, and an intrinsic yielding rate $\dot{N}$ can be defined as

$$
\dot{N}=-\frac{1}{F} \frac{d F}{d t}
$$

Suppose that the applied stress is increased linearly with time $t$ at a constant rate $\dot{\sigma}$, i.e. $\sigma=\dot{\sigma} t$, $F(\sigma)$ becomes

$$
F(\sigma)=\exp \left[-\frac{1}{\dot{\sigma}} \int_{0}^{\sigma} \dot{N}\left(\sigma^{\prime}\right) d \sigma^{\prime}\right] \text { or } \quad \int_{0}^{\sigma} \dot{N}\left(\sigma^{\prime}\right) d \sigma^{\prime}=\dot{\sigma} \ln (1 / F)
$$

Next, suppose that, as said before, the yield strength is controlled by Taylor interactions within the initial dislocation network of the specimen (see Figure 1 for examples). This has been found to be the case from dislocation dynamics simulations of micro-pillar compression in the non-starved state (Rao et al., 2008; Rao et al., 2007), and should be a rather weak assumption. 
Thus, the stress required to mobilize a dislocation segment of free length $L$ within the mesh network is

$$
\sigma_{s} \approx \mu^{\prime} b / L,
$$

where $b$ is Burgers vector and $\mu^{\prime}$ is the shear modulus normalized by the Schmid factor of the most favourable slip system, which should be a constant in the ensemble since all replicas should have the same orientation. If the initial dislocation structure is not uniform but the mesh lengths exhibit a probability distribution $p_{L}(L)$, then, through equation (4), this corresponds to a distribution $p_{s}\left(\sigma_{s}\right)$ of the resistance $\sigma_{s}$ given by

$$
p_{s}\left(\sigma_{s}\right)=p_{L}(L) \times\left|d L / d \sigma_{s}\right|=\frac{\mu \prime b}{\sigma_{s}{ }^{2}} \times p_{L}(L) .
$$

Equation (2) above gives the yielding rate $\dot{N}$ as observed from the rate at which the samples in the ensemble yield one after another as time proceeds, but $\dot{N}$ is obviously related to whether dislocations in the representative microstructure of the specimens can have substantial movement under the instantaneous stress $\sigma$. In the latter "microscopic" view, $\dot{N}$ is simply the probability that a specimen randomly drawn from the ensemble will yield in the next unit time interval, given its microstructure and the current stress level. From this viewpoint, $\dot{N}$ can be expected to scale with (i) the average quantity of mobile dislocation segments in the randomly drawn sample, and (ii) the average velocity $v$ of such mobile dislocations. The dislocation velocity counts here because if the dislocations move very slowly in free flight even if they can be freed out from the mesh structure, they may not produce enough slip which would lead to macroscopically observable yielding in the next time interval. For the mobile dislocation quantity in (i) above, imagine that the sampling process is one of randomly picking a volume $D^{3}$ out of an infinitely large microstructure describable by the mesh-length probability $p_{L}(L)$ in equation (5). A situation pertinent to the experiments to be described later in Section 3 is that a number of micro-specimens, each with a volume $D^{3}$, are milled out from a very large grain with a certain initial dislocation microstructure describable by $p_{L}(L)$. The average amount of mobile dislocation segments in a random sample taken from the master microstructure should therefore scale with its volume $D^{3}$, as well as the overall probability of encountering such mobile segments in the infinite master microstructure, i.e. 


$$
D^{3} \times P\left(\sigma_{s}<\sigma\right)=D^{3} \times \int_{0}^{\sigma} p_{s}\left(\sigma_{s}^{\prime}\right) d \sigma_{s}^{\prime}=D^{3} \times \int_{0}^{\sigma} \frac{p_{L}\left(L=\mu \prime b / \sigma_{s}^{\prime}\right)}{\sigma_{s}^{\prime 2}} d \sigma_{s}{ }^{\prime}
$$

The yielding rate $\dot{N}$ is therefore expected to scale as

$$
\dot{N} \propto v \times D^{3} \times P\left(\sigma_{s}<\sigma\right) \propto \sigma^{n} \times D^{3} \times \int_{0}^{\sigma} \frac{p_{L}\left(L=\mu \prime b / \sigma_{s}^{\prime}\right)}{{\sigma_{s}^{\prime}}^{2}} d \sigma_{s}^{\prime}
$$

where the average velocity of the mobile dislocations $v$ is taken to be of the usual power-law form $v \propto \sigma^{n}$. Substituting equation (7) into the second relation in equation (3) gives

$$
\int_{0}^{\sigma}\left(\sigma^{\prime n} \int_{0}^{\sigma^{\prime}} \frac{p_{L}\left(L=\mu \prime b / \sigma_{s}^{\prime}\right)}{\sigma_{s}^{\prime 2}} d \sigma_{s}^{\prime}\right) d \sigma^{\prime} \propto D^{-3} \dot{\sigma} \ln (1 / F)
$$

Equation (8) governs the magnitude of $\sigma$ needed to achieve a given survival probability, say, $F=$ $50 \%$, for a given sample size $D$. For different sample sizes $D$ the required $\sigma$ will be different, and if we demand the resultant $\sigma(D)$ relation to be the power law in equation (1), equation (8) then becomes

$$
\int_{0}^{\sigma}\left(\sigma^{\prime n} \int_{0}^{\sigma^{\prime}} \frac{p_{L}\left(L=\mu \prime b / \sigma_{s}^{\prime}\right)}{{\sigma_{s}^{\prime}}^{2}} d \sigma_{s}{ }^{\prime}\right) d \sigma^{\prime} \propto \sigma^{3 / m} \dot{\sigma} \ln (1 / F)
$$

Differentiating equation (9) with respect to $\sigma$ twice leads to the condition

$$
p_{L}\left(L=\mu^{\prime} b / \sigma\right) \propto \sigma^{\left(\frac{3}{m}-n\right)} \dot{\sigma} \ln (1 / F) \propto L^{-\left(\frac{3}{m}-n\right)},
$$

which indicates that for equation (1) to be valid, the mesh-length distribution in the initial dislocation network has to be a power law $p_{L}(L) \propto L^{-q}$ with $q$ given by

$$
m=\frac{3}{q+n}
$$

Reversibility of the proof - Equations (9) to (11) above show that if the size dependence of strength is the power law $\sigma \sim D^{-m}$, then the mesh-length distribution must be the power law $p_{L}(L) \propto L^{-q}$ with $q$ given by equation (11). As has been shown in ref. (Ngan, 2011), we can equally first assume that the dislocation mesh-length distribution is the power law $p_{L}(L) \propto L^{-q}$ and substitute this into equation (7), and then substitute the resultant $\dot{N}$ into equation (3) to prove that the size dependence of strength must be the power law $\sigma \sim D^{-m}$, with $m$ given by equation 
(11). Thus, the proof is reversible: within the assumptions of the present model, a one-to-one correspondence exists between a power-law size dependence of strength, and a power-law distribution of the initial mesh lengths. From the discussion above, the assumptions of the present model can be explicitly summarized as: (i) Taylor interactions within the initial dislocation network determine yield strength, (ii) the ensemble yielding rate $\dot{N}$ scales with the probability for the given specimen size to contain mobile dislocation segments and their velocity, c.f. equation (7), and (iii) the dislocation velocity obeys a power law $v \propto \sigma^{n}$ with stress. All these are rather weak assumptions for a wide range of metals.

\subsection{Sampling from 2-D projections of 3-D dislocation networks}

Although equation (11) predicts that the power-law exponent $m$ of the size dependence of strength is inversely related to the exponent $q$ of the mesh-length distribution, actual experimental verification is difficult since the $q$ involved is for the mesh-length distribution in 3$\mathrm{D}$ but the initial dislocation pattern can only be conveniently imaged in the transmission electron microscope (TEM) as 2-D projections. Even if the TEM tomography technique is used, the sampling is still from thin slices of the 3-D sample, and so is still based on 2-D. Zaiser et al. (Zaiser et al., 1999) assumed that the fractal dimension of a 3-D dislocation network is that of its 2-D projection plus one, but more recent work has shown that there is no general relation between the dimensions of a 3-D fractal and its 2-D projections (Akkari et al., 2008). Here, in order to proceed, we take a simplification step by arguing that, instead of equation (7), the ensemble yielding rate $\dot{N}$ can be estimated by sampling from a 2-D projection of the network. This amounts to saying that the samples are now 2-D with area $D^{2}$. The average amount of mobile dislocation segments in such a random sample will be

$$
D^{2} \times \int_{0}^{\sigma} \frac{p_{2-D}\left(L=\mu^{\prime} b / \sigma_{s}^{\prime}\right)}{\sigma_{s}^{\prime 2}} d \sigma_{s}^{\prime}
$$

where $p_{2-D}(L)$ is the mesh-length distribution sampled from a 2-D projection of the 3-D master microstructure, and $D^{2}$ the projected area of the sample. Then, in lieu of equation (7), the yielding rate $\dot{N}$ now scales as 


$$
\dot{N} \propto \sigma^{n} \times D^{2} \times \int_{0}^{\sigma} \frac{p_{2-D}\left(L=\mu \prime b / \sigma_{s}^{\prime}\right)}{\sigma_{s}^{\prime 2}} d \sigma_{s}^{\prime}
$$

and following operations similar to those in equations (8) to (10), we obtain

$$
p_{2-D}(L) \propto L^{-q^{\prime}}
$$

where $q^{\prime}=2 / m^{\prime}-n$, or

$$
m^{\prime}=\frac{2}{q^{\prime}+n}
$$

and $m^{\prime}$ is the exponent of the size dependence of strength of such 2-D samples, i.e. $\sigma \sim D^{-m^{\prime}}$. With equation (14), the yielding rate $\dot{N}$ in equation (12) becomes

$$
\dot{N}=\alpha D^{2} \sigma^{q^{\prime}+n-1} /\left(q^{\prime}-1\right)
$$

where $\alpha$ is a constant, and then the survival probability $F(\sigma)$ in equation (3) can be integrated to become

$$
F(\sigma)=\exp \left[-\frac{\alpha}{\sigma\left(q^{\prime}+n\right)\left(q^{\prime}-1\right)} D^{2} \sigma^{q^{\prime}+n}\right]
$$

\section{Experimental Procedures}

To verify the key predictions above, namely, that a power-law dependence of strength on size corresponds to a power-law distribution of the mesh lengths in the initial dislocation network, and that the power exponent $m$ for strength is inversely related to the power exponent $\Lambda$ for the dislocation network, 99.9999 wt. \% pure aluminum pieces were annealed or cold-rolled to $7 \%$ or $15 \%$, in order to achieve different initial dislocation networks. Micro-pillars were machined from these different pre-strained states and were compression tested in a flat-punch nanoindenter. The detailed experimental procedures are as follows.

A round piece of aluminum bulk was cut from an ultrapure (> 99.9999 wt. \% pure) aluminum rod. After annealing treatment in an air furnace at $500{ }^{\circ} \mathrm{C}$ for 24 hours, the sample was mechanically polished and then electro-polished in a solution of perchloric acid and methanol in 1:9 volume ratio at $-30^{\circ} \mathrm{C}$ for around 3.5 minutes. The size and orientation of crystals in the bulk 
were characterized by electron back-scattered diffraction (EBSD) in a Hitachi S4800 FEG scanning electron microscope (SEM). A large grain with orientation [516] and diameter $>2 \mathrm{~mm}$ was chosen for the subsequent experiments. The bulk yield strength of the gain was estimated as one-third of the Vickers hardness measured by micro-indentation.

Circular micro-pillars were fabricated from the chosen grain by a Quanta 200 3D Dual Beam FIB/SEM focused-ion beam (FIB) system. The milling operation was performed with an ion current of 20nA for the outermost coarse milling, and a small current of 50pA for the final milling, at a voltage at $30 \mathrm{kV}$. Three typical sizes of micro-pillars were fabricated in this study, namely, $5.6 \mu \mathrm{m}, 3.3 \mu \mathrm{m}$ and $1.2 \mu \mathrm{m}$ diameter with aspect ratio around 4:1. The actual dimensions of the micro-pillars were determined from imaging with a LEO1530 SEM, and were used in the analysis of the results. Typically, around 10 pillars of each size group were compression tested in each condition, in order to understand the spread of the data. The uniaxial compression tests were carried out in a G200 Nanoindenter with a diamond flat punch at room temperature. The flat punch was manufactured from a diamond Berkovich tip by FIB milling, and has diameter and height of about 8.5 and $1.5 \mu \mathrm{m}$ respectively. The compression tests were performed in a load

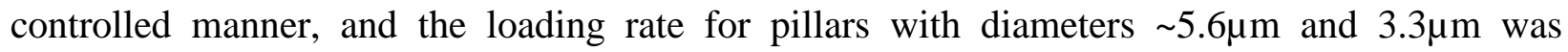
$40 \mu \mathrm{N} / \mathrm{s}$, and was $10 \mu \mathrm{N} / \mathrm{s}$ for the smaller pillars of $\sim 1.2 \mu \mathrm{m}$ diameter. During the tests, the flat punch was made to approach the specimen at a very slow speed until it was very close to the specimen surface. After thermal fluctuations have settled to the minimum requirement, the punch was made to reach the top of the pillar and load was applied linearly with time to the maximum magnitude before unloading. The stress-strain data during compression were obtained and the deformed appearances of the pillars were characterized by SEM afterwards.

After compression and characterization of the pillars fabricated on the annealed bulk, the bulk sample was cold rolled to 7\% reduction. Fresh micro-pillars were fabricated on the same grain which has now been pre-strained, and these were compressed and analyzed using the same procedures as above. Afterwards, the bulk sample was further cold-rolled to an accumulated 15\% reduction, and same processes were repeated. This way, the effects of different pre-strains can be studied on the same grain so that the data are not affected by different crystal orientation.

To investigate the typical initial dislocation microstructures at different pre-strains, TEM examination was carried out. Slices were cut from the annealed and subsequently deformed bulk 
sample, albeit not from the same grain from which the micro-pillars were made. Although they were not cut from the same grain, the dislocation structures they contained should be representative of the different pre-strain values. These slices were initially mechanically polished on both sides until the average thickness was reduced to about $150 \mu \mathrm{m}$. These were then punched into standard 3mm diameter discs, which were electro-polished by the twin-jet method with the same electrolyte and conditions as mentioned above. TEM examination was carried out on a Philips Tecnai microscope operated at 200kV. Montages of digitally recorded TEM images were processed for fractal analysis. The image processing procedure included two steps: (1) with an image processor, the contrast was increased to sharpen the dislocation lines in the image; (2) the image was converted into binary format by the MATLAB software, based a certain threshold of grayscale. After this procedure the pixels in the image are either black if they fall on dislocation lines or cellular walls, or white if they fall in dislocation-free regions.

\section{Experimental Results}

Figure 1 shows the typical TEM dislocation structures at different pre-strains, as well as the digitized bitmaps of the dislocation images. It is clear that in the annealed state, the dislocation density was low on the order of $10^{11} \mathrm{~m}^{-2}$. At $7 \%$ reduction, the dislocation density increased but significant cell formation also occurred. Cell formation was more severe at $15 \%$ reduction, with more refined cell size than the $7 \%$ reduction case. The cell structure in the deformed state makes the dislocation distribution highly inhomogeneous.

The bitmap images shown in Figure 1 were analyzed by the box-counting method (Zaiser et al., 1999). To do this, each bitmap image was covered by successive sets of grids with decreasing side length $\Delta x$, and the number of boxes containing at least one dislocation segment $N(\Delta x)$ was counted for each grid size. Figure 2 shows that for all the three cases of $0 \%, 7 \%$ and $15 \%$ pre-strain, $N(\Delta x)$ decreases with $\Delta x$ approximately as a power law

$$
N(\Delta x) \propto \Delta x^{-\Lambda_{B}},
$$


where $\Lambda_{B}$, known as the box-counting dimension, is an estimate of the fractal dimension of the dislocation pattern. The probability density distribution of $\Delta x$ is $p(\Delta x) \propto \frac{d N(\Delta x)}{d(\Delta x)} \propto(\Delta x)^{-\left(\Lambda_{B}+1\right)}$, and when compared to equation (13), $q^{\prime}$ can be estimated as

$$
q^{\prime} \approx \Lambda_{B}+1
$$

However, it must be remembered that $\left(\Lambda_{B}+1\right)$ is not really the same as $q^{\prime}$ which is the exponent in the mesh-length distribution. To measure $q^{\prime}$ directly would be difficult since the mesh points, i.e. the dislocation nodes, cannot be identified unambiguously from the TEM images, and without knowing the mesh points, the free segment lengths $L$ in the mesh cannot be identified. Although box-counting dimension $\Lambda_{B}$ is not identical to $\left(q^{\prime}-1\right)$, it is nevertheless a measure which can be obtained rather unambiguously from a digitized pattern. The $\Lambda_{B}$ values were measured as the slopes of the curves in Figure 2 over the $\Delta x$ range from 0.1 to $1 \mu \mathrm{m}$, since this range would represent a reasonable internal length scale of the dislocation microstructure considering the sizes of the present micro-pillars. The measured values of $\Lambda_{B}$ are $1.52 \pm 0.02$ $1.62 \pm 0.02$ and $1.78 \pm 0.01$ for the $0 \%, 7 \%$ and $15 \%$ pre-strains respectively. In addition to the box-counting dimensions, the mass dimensions $\Lambda_{M}$ (Zaiser et al., 1999) of the bitmap images were also determined and the results are shown in Figure 2(b). Here, $M(r)$ is the average number of dislocation segments within a circle of radius $r$ around another randomly chosen dislocation segment, and $M(r)$ increases with $r$ as a power law in a certain range with an exponent $\Lambda_{M}$, i.e. $M(r) \propto r^{\Lambda_{M}}$. In Figure 2(b) $C$ is a normalization constant enabling $M(r)=1$ when $r=1 \mu m$. For the $0 \%, 7 \%$ and $15 \%$ pre-strained samples $\Lambda_{M}$ are $1.56 \pm 0.011 .69 \pm 0.01$ and $1.80 \pm$ 0.01 respectively, which are consistent with the box-counting dimensions.

The load-displacement plots of the micro-pillars' compression tests were jerky and the compressed appearances in the SEM exhibited discrete slip steps on the pillars' surface as similar to previously observed (Ng and Ngan, 2008a, b), and due to the limitation of space these results are not shown here. The yield strength of each compressed pillar was determined as the nominal direct stress at which the first strain burst occurred, which was taken as the maximum fluctuation in strain in the initial elastic-like loading regime. The noise floor of the machine is known to be a limiting factor for the accurate determination of very small strain bursts (Ng and Ngan, 2008b; 
Zaiser et al., 2008), and considering of this factor for the present machine led to the conclusion that strain bursts larger than $\sim 2 \times 10^{-4}$ could be reliably identified. Figure 3 shows the yield strength versus size of micro-pillars with $0 \%, 7 \%$ and $15 \%$ pre-strain respectively. It can be seen that at sizes $5.6 \mu \mathrm{m}$ and $3.3 \mu \mathrm{m}$, the pristine pillars were substantially weaker than the prestrained pillars, but at the smallest size of $1.2 \mu \mathrm{m}$ tested, the pristine pillars were marginally stronger than their pre-strained counterparts. The strengths of the pre-strained pillars also scatter more as size decreases, than the pristine pillars - for instance, the strength of the $7 \%$ pre-strained pillars with diameter $\sim 1.2 \mu \mathrm{m}$ scattered from $50 \mathrm{MPa}$ to $180 \mathrm{MPa}$. This correlates well with the cellular dislocation distribution in the pre-strained cases as shown in Figure 1, where cell sizes can be several microns large. Thus, if different micron-sized samples are sampled (FIB-milled) from such a microstructure, some will contain dense dislocation walls, and others will contain a lot of dislocation-free spaces, and so it is not surprising that the strength data exhibit large scatter. It is exactly this nature of scattering that the model developed in Section 2 above becomes pertinent, as later analyses will show.

As can be seen from Figure 3, for all the three size groups of specimens, their yield strength decreases with diameter approximately as a power-law $\sigma \propto D^{-m}$, and the exponent $m$ decreases with the magnitude of the pre-strain, from $m \sim 0.98 \pm 0.08$ for the pristine case, to $0.62 \pm 0.09$ and $0.51 \pm 0.09$ for the $7 \%$ and $15 \%$ pre-strained case respectively. The values of errors here are the standard errors of the parameter, and $R$-squared correlation coefficients for the three cases are $0.90,0.59$ and 0.77 respectively. Due to the scatter of data as discussed above, the uncertainties in the $m$ values are not small, but the trend that $m$ decreases as pre-strain increases is very clear. Together with Figure 2, the results now can verify the trend predicted by equation (14) - as pre-strain increases, $\Lambda_{B}$ increases, but $m$ decreases, so there is an inverse relation between $m$ and the exponent of the mesh-length distribution.

\section{Analysis of Results}

The loading rate used for the pillar compression, namely, $40 \mu \mathrm{N} / \mathrm{s}$ for $\sim 3.3 \mu \mathrm{m}$ and $5.6 \mu \mathrm{m}$ pillar diameter and $10 \mu \mathrm{N} / \mathrm{s} \sim 1.2 \mu \mathrm{m}$ diameter, were chosen to ensure that the test data can be logged at comparable rates and that the average strain rate was on the order of $10^{-3} \mathrm{~s}^{-1}$, but they did not correspond to a fixed stress rate $\dot{\sigma}$ because the pillar diameters were different. However, 
it was found that, with such choices of the loading rate, the stress rate $\dot{\sigma}$ varied with diameter $D$ in roughly a power law $\dot{\sigma} \propto D^{-k}$, where $k=0.84 \pm 0.25$, and substituting this into equation (8) and carrying out similar operations afterwards, it can be shown that, for 2-D samples,

$$
m^{\prime}=\frac{2+k}{q^{\prime+n}}
$$

Thus, the basic form of the inverse relation between $m^{\prime}$ and $q^{\prime}$ is unchanged c.f. equation (14). Figure 4 shows the plot of $m$ vs $\Lambda_{B}$ determined from the experiments, and indeed an inverse relation exists. However, if $\left(\Lambda_{B}+1\right)$ is taken as $q^{\prime}$ and $m$ taken as $m^{\prime}$, and the trend line in Figure 4 is compared with equation (17), $n$ would turn out to be a negative value. This indicates that $\left(\Lambda_{B}+1\right)$ is not identical to $q^{\prime}$, and $m$ not identical to $m^{\prime}$. In spite of this, if $n$ is taken to be 1.5 and $q^{\prime}$ is taken to be the experimental values of $\left(\Lambda_{B}+1\right)$, equation (17) would yield $m^{\prime}=$ $0.71 \pm 0.06,0.69 \pm 0.06$ and $0.66 \pm 0.06$ respectively for $0 \%, 7 \%$ and $15 \%$ pre-strain. The error bars in $m^{\prime}$ here are the latter times the square-root of the sum of the squares of the relative errors of all the terms in equation (17). The $m^{\prime}$ values for the $7 \%$ and $15 \%$ prestrained cases calculated from eqn. (17) are plotted in Figure 4, and although not as strong as that observed experimentally, the calculated $m^{\prime}$ data still exhibit a decreasing trend on increasing pre-straining, and perhaps the deviation from the experimental trend for $m$ is due to the 2-D vs 3-D considerations discussed above.

Next, we consider the spread of the strength data by referencing to equation (15). For each pillar size group and pre-straining condition, the spread of the data in Figure 3 is converted into $F(\sigma)$ data points in Figure 5(a,b), for the $7 \%$ and $15 \%$ pre-strained cases respectively (the pristine case will be dealt with in the next Section). To do this, the strength data for each group are ranked in ascending order, and the survival probability for each strength data is evaluated as $F=1-i / N$, where $N$ is the total number of strength data in the group, and $i$ is the rank. The $F$ values are plotted vs the strength value $\sigma$ as discrete points in Figure 5(a,b). These trends for different pillar sizes are fitted with the $F(\sigma)$ relation predicted in equation (15) by choosing suitable values for $\alpha$ and $n$, while $q^{\prime}$ is chosen to be close to the estimate $\left(\Lambda_{B}+1\right)$ from the experimentally observed fractal dimension for either pre-strain value. It was found that by choosing $n=1.5, \alpha=2.0 \times 10^{-6} \mathrm{~s}^{-1} \mu \mathrm{m}^{-2}(\mathrm{MPa})^{-3.1}$ and $q^{\prime}=2.6 \pm 0.2$, equation (15) would produce reasonably good fit to the experimental $F(\sigma)$ data for all the three pillar sizes in the $7 \%$ 
pre-strained case (Figure 5(a)). For the 15\% pre-strained case shown in Figure 5(b), reasonably good fit is achieved by choosing $n=1.5, \alpha=5.0 \times 10^{-8} \mathrm{~s}^{-1} \mu \mathrm{m}^{-2}(\mathrm{MPa})^{-3.3}$ and $q^{\prime}=2.8 \pm$ 0.2 . The fact that in each pre-strained case, the experimental $F(\sigma)$ data for all three pillar size groups can be fitted to equation (15) by choosing the same set of $n, \alpha$ and $q^{\prime}$ values help verify the theory, as these parameters are not expected to change for different sample sizes.

We also carried out analysis for the $0 \%$ prestrained case using eqn. (17) from the fractal model, but the match with the experimental data is not that satisfactory. This is not surprising since Figure 2 shows that for the $0 \%$ prestrained case, the double-logarithmic plot of $N(\Delta x)$ is not quite linear and so the fractal model should not be accurate. The theoretical reversibility argument proofed in Section 2 states that if the initial dislocation mesh-length distribution is not strictly a power law, the size dependence of strength should also be not an absolute power law, and considering the scatter of the strength data in Figure 3, this possibility should not be eliminated. In spite of this, if the true, non-fractal mesh-length distribution $p_{2-D}(L)$ is measured from the TEM micrographs, the correct size dependence of strength, together with the spread of the data, can still be worked out from equations (3) and (12). In the Appendix, such an analysis is carried out for the case of $0 \%$ prestrain. With the experimental $N(\Delta x)$ plot from Fig. 2 for the $0 \%$ prestrained case, the analysis predicts that while the $\sigma-D$ relation is not an absolute power-law, it is still well approximated by a power law with an approximate $m^{\prime}$ value of $0.89 \pm 0.08$, which is also plotted in Figure 4 and is close to the experimental value.

\section{Discussion}

The present work shows that in the micro-regime, there is a one-to-one correspondence between the fractal distribution of the initial dislocation network, and the power-law size effect of strength. Furthermore, if the initial dislocation network is not strictly fractal, the size effect may still be approximately power-law. However, such a conclusion is expected to hold for a finite range of specimen sizes only, possibly in the regime from a micron to tens of microns regime. First, submicron- or nano-sized specimens taken from a master microstructure with a mild dislocation density should contain very few, or even no initial dislocation segments. In such cases, strength cannot be controlled by Taylor interactions but more likely by nucleation of 
dislocations from specimen surfaces (Zuo and Ngan, 2006; Mordehai et al. 2011). We have recently demonstrated experimentally that the strength of Al pillars $\sim 6 \mu \mathrm{m}$ large is proportional to the square-root of the residual dislocation density after deformation, but for $\sim 1 \mu \mathrm{m}$ pillars, there is no correlation between strength and the residual dislocation density, which remains at a low initial value (Gu and Ngan, 2012). This shows that while the Taylor mechanism works for specimens that are several microns large, it breaks down for micron-sized specimens.

On the other hand, for very large specimen sizes $D$, strength should tend to a constant bulk value $\sigma_{\text {bulk }}$, instead of to zero according to the power-law $\sigma \sim D^{-m}$. While the transition from the $\sigma \sim D^{-m}$ behavior to the bulk behavior $\sigma \rightarrow \sigma_{\text {bulk }}$ is still an open area warranting further investigations, a number of factors are likely to be contributing. First, Hähner and Zaiser, 1999, also assumed that strength is determined by Taylor interactions, but they obtained a size independent macroscopic flow stress because they made the additional assumption that compatibility stresses exist between different volumes of the deforming crystal. Secondly, the fractal law $p_{L}(L) \propto L^{-q}$ for the distribution of the mesh lengths may not hold for indefinitely long lengths $L$, and for very large $L, p_{L}(L)$ drops much faster towards zero than the power law. This amounts to saying that very long free segments of dislocations may not be found in bulk specimens according to the power-law distribution, and there could even be some maximum cutoff value for $L$ which controls the bulk strength $\sigma_{\text {bulk }}$. A third possibility more pertinent for impurity-containing specimens is that the power law can still describe the long dislocations, but in the bulk condition the Taylor interaction becomes so weak for these long dislocations that some other mechanism, such as solute pinning, takes over and control strength.

\section{Conclusions}

In this study, an explanation for the power-law size effect on the strength of microcrystals with existing dislocations has been developed. A theoretical analysis assuming that yield strength is determined by Taylor interactions within the initial dislocation network has shown that if the size dependence of strength is a power law, then the initial mesh-length distribution must also be a power law, and vice versa. Moreover, the power exponent of the strength-size relation and that of the mesh-length distribution are predicted to be inversely related. 
Experiments on ultrapure aluminum with different pre-straining showed that the resultant dislocation patterns were indeed approximate fractals, the dimensions of which were found to relate inversely with the power exponents of the strength-size relation as determined from compression tests on micro-pillars fabricated from the different pre-strained states.

\section{Appendix - Analysis for Non-power-law Distributions}

The analysis leading to equation (15) was based on the premise that the initial meshlength distribution is a power law. In Figure 2, the double-logarithmic $N(\Delta x)$ plot for the pristine case ( $0 \%$ prestraining) exhibits some degree of curvature, and so as an illustrative exercise, we show here that non-fractal dislocation patterns can also be dealt with using the present theoretical framework.

Thus, instead of using a power law to represent the $N(\Delta x)$ relation, the probability density of mesh lengths $L$ can be worked out as

$$
p_{2-D}(L) \propto \frac{d N(L)}{d L} \quad \text { or } \quad p_{2-D}(L) \propto \frac{d N(\Delta x)}{d(\Delta x)} .
$$

In this case, for 2-D sampling, the survival probability is given from equations (3) and (12) as

$$
F(\sigma)=\exp \left[-\frac{\alpha D^{2}}{\dot{\sigma}} \int_{0}^{\sigma}\left(\sigma^{\prime n} \int_{0}^{\sigma^{\prime}} p_{s}\left(\sigma_{s}{ }^{\prime}\right) d \sigma_{s}{ }^{\prime}\right) d \sigma^{\prime}\right],
$$

and the size dependence of strength for a given $F$ as

$$
\frac{D^{2}}{\dot{\sigma}} \int_{0}^{\sigma}\left({\sigma^{\prime \prime}}^{n} \int_{0}^{\sigma^{\prime}} p_{s}\left(\sigma_{s}{ }^{\prime}\right) d \sigma_{s}{ }^{\prime}\right) d \sigma^{\prime}=\frac{1}{\alpha} \ln (1 / F),
$$

where $\alpha$ is a constant.

The $N(\Delta x)$ relation for the pristine case can be fitted accurately by the equation,

$$
N(\Delta x)=\frac{1}{2.24 \times 10^{-4}+1.16 \times 10^{-2} \Delta x^{1.80}}
$$

within the range from 0.1 to $1 \mu \mathrm{m}$, as shown in Figure 6(a). From equation (18) the probability density of mesh lengths can be estimated as 


$$
p_{2-D}(L)=\frac{3.48 E \times 10^{-2} L^{0.80}}{\left(2.24 \times 10^{-2}+1.16 L^{1.80}\right)^{2}} .
$$

Figure 6(b) shows the plot of $p_{2-D}(L)$. For aluminum single crystal with orientation of [516], the shear modulus $\mu=32 \mathrm{GPa}$ and Burgers vector $b=2.8 \times 10^{-4} \mu \mathrm{m}$, and so from equation (5) the probability distribution of $\sigma_{s}$ is

$$
p\left(\sigma_{S}\right) \propto \frac{\sigma_{S}^{-2.80}}{\left(1+2681 \sigma_{S}^{-1.80}\right)^{2}} .
$$

The survival probability in Eq. (19) therefore becomes

$$
F(\sigma)=\exp \left[-\frac{\beta D^{2}}{\dot{\sigma}} Y(\sigma)\right]
$$

where

$$
Y(\sigma)=\int_{0}^{\sigma} \frac{\sigma^{n}}{1+2681 \sigma^{-1.80}} d \sigma^{\prime}
$$

and $\beta$ is a constant. $Y(\sigma)$ can be calculated by numerical integration with $n$ set at 1.5 in accordance with the previous results, and the result is shown in Figure 6(c) in double logarithmic scale. It can be seen that for $\sigma$ values between 20 to $170 \mathrm{MPa}, Y(\sigma)$ can be approximately described by a power-law equation

$$
Y(\sigma) \propto \sigma^{a}
$$

with $a=3.20 \pm 0.01$. Therefore the survival probability in equation (21) can be expressed as

$$
F(\sigma)=\exp \left[-\frac{1}{\dot{\sigma}} \beta^{\prime} D^{2} \sigma^{a}\right]
$$

where $\beta^{\prime}$ is a constant. As discussed before, the loading rates used give rise to $\dot{\sigma} \propto D^{-0.84}$, and so a fixed $F$ corresponds to $D^{2.84} \sigma^{3.2}=$ constant, or $\sigma \propto D^{-0.89}$. Hence, with this refined analysis, the predicted exponent $m^{\prime}$ for size dependence of strength in the pristine case is $0.89 \pm 0.08$, which is close to the experimental value of $0.98 \pm 0.08$ in Figure 3. This data point is also shown in Figure 4. Figure 7 shows the theoretical survival probability $F(\sigma)$ computed from equation (22) with $\beta^{\prime}$ set at $1.5 \times 10^{-6} \mathrm{~s}^{-1} \mu \mathrm{m}^{-2}(\mathrm{MPa})^{-2.2}$ and $a=3.2 \pm 0.1$. It can be seen that with such choice of parameters, the theoretical curves fit the experimental data well. 


\section{Acknowledgements}

The work described in this paper was supported by grants from the Research Grants Council (Project No. 7159/10E) as well as from the University Grants Committee (Project No. SEG-HKU06) of the Hong Kong Special Administrative Region.

\section{References}

Akkari, H., Bhouri, I., Dubois, P., Bedoui, M.H., 2008. On the Relations Between 2D and 3D Fractal Dimensions: Theoretical Approach and Clinical Application in Bone Imaging. Math. Model. Nat. Phenom. 3, 48-75.

Brenner, S.S., 1956a. The mechanism of densification during sintering of metallic particles. Acta Metall. 4, 58-61.

Brenner, S.S., 1956b. Tensile Strength of Whiskers. J. Appl. Phys. 27, 1484-1492.

Brenner, S.S., 1957. Plastic Deformation of Copper and Silver Whiskers. J. Appl. Phys. 28, 1023-1027.

Dimiduk, D.M., Uchic, M.D., Parthasarathy, T.A., 2005. Size-affected single-slip behavior of pure nickel microcrystals. Acta Mater. 53, 4065-4077.

Dimiduk, D.M., Uchic, M.D., Rao, S.I., Woodward, C., Parthasarathy, T.A., 2007. Overview of experiments on microcrystal plasticity in FCC-derivative materials: selected challenges for modelling and simulation of plasticity. Modell. Simul. Mater. Sci. Eng. 15, 135-146.

Dou, R., Derby, B., 2009. A universal scaling law for the strength of metal micropillars and nanowires. Scripta Mater. 61, 524-527.

El-Awady, J.A., Wen, M., Ghoniem, N.M., 2009. The role of the weakest-link mechanism in controlling the plasticity of micropillars. J. Mech. Phys. Solids 57, 32-50.

Frick, C.P., Clark, B.G., Orso, S., Schneider, A.S., Arzt, E., 2008. Size effect on strength and strain hardening of small-scale 111 nickel compression pillars. Mat. Sci. Eng. A-Struct. 489, 319-329.

Greer, J.R., Oliver, W.C., Nix, W.D., 2005. Size dependence of mechanical properties of gold at the micron scale in the absence of strain gradients. Acta Mater. 53, 1821-1830. 
Greer, J.R., De Hosson, J.T.M., 2011. Plasticity in small-sized metallic systems: Intrinsic versus extrinsic size effect. Prog. Mater. Sci. 56, 654-724.

Greer, J.R., Nix, W.D., 2006. Nanoscale gold pillars strengthened through dislocation starvation. Phys. Rev. B 73, 245410.

Greer, J.R., Oliver, W.C., Nix, W.D., 2005. Size dependence of mechanical properties of gold at the micron scale in the absence of strain gradients. Acta Mater. 53, 1821-1830.

Gu, R., A.H.W. Ngan. Effects of pre-straining and coating on plastic deformation of aluminum micro-pillars, accepted for publication in Acta Materialia.

Hähner, P, Zaiser, M., 1999. Dislocation dynamics and work hardening of fractal dislocation cell structures. Mater. Sci. Eng. A272, 443-54.

Han, S.M., Bozorg-Grayeli, T., Groyes, J.R., Nix, W.D., 2010. Size effects on strength and plasticity of vanadium nanopillars. Scripta Mater. 63, 1153-1156.

Huang, Y., Gao, H., Nix, W.D., Hutchinson, J.W., 2000a. Mechanism-based strain gradient plasticity - II. Analysis. J. Mech. Phys. Solids 48, 99-128.

Huang, Y., Xue, Z., Gao, H., Nix, W.D., Xia, Z.C., 2000b. A study of microindentation hardness tests by mechanism-based strain gradient plasticity. J. Mater. Res. 15, 1786-1796.

Kim, J.-Y., Greer, J.R., 2009. Tensile and compressive behavior of gold and molybdenum single crystals at the nano-scale. Acta Mater. 57, 5245-5253.

Kim, J.-Y., Jong, D., Greer, J.R., 2010. Tensile and compressive behavior of tungsten, molybdenum, tantalum and niobium at the nanoscale. Acta Mater. 58, 2355-2363.

Kunz, A., Pathak, S., Greer, J.R., 2011. Size effects in Al nanopillars: single crystalline vs. bicrystalline. Acta Mater. 59, 4416-4424.

Lee, S.-W., Han, S.M., Nix, W.D., 2009. Uniaxial compression of fcc Au nanopillars on an MgO substrate: The effects of prestraining and annealing. Acta Mater. 57, 4404-4415.

Maaß, R., Meza, L., Gan, B., Tin, S., Greer, J.R., 2012. Ultrahigh Strength of Dislocation-Free Ni3Al Nanocubes. Small 8, 1869-1875.

Mordehai, D., Lee, S-K., Backes, B., Srolovitz, D.J., Nix, W.D., Rabkin, E., 2011. Size effect in compression of single-crystal gold microparticles. Acta Mater. 59, 5202-5215.

Ng, K.S., Ngan, A.H.W., 2008a. Stochastic nature of plasticity of aluminum micro-pillars. Acta Mater. 56, 1712-1720. 
Ng, K.S., Ngan, A.H.W., 2008b. Stochastic theory for jerky deformation in small crystal volumes with pre-existing dislocations. Philos. Mag. 88, 677-688.

Ngan, A.H.W., 2011. An explanation for the power-law scaling of size effect on strength in micro-specimens. Scripta Mater. 65, 978-981.

Nix, W.D., Gao, H.J., 1998. Indentation size effects in crystalline materials: A law for strain gradient plasticity. J. Mech. Phys. Solids 46, 411-425.

Norfleet, D.M., Dimiduk, D.M., Polasik, S.J., Uchic, M.D., Mills, M.J., 2008. Dislocation structures and their relationship to strength in deformed nickel microcrystals. Acta Mater. 56, 2988-3001.

Parthasarathy, T.A., Rao, S.I., Dimiduk, D.M., Uchic, M.D., Trinkle, D.R., 2007. Contribution to size effect of yield strength from the stochastics of dislocation source lengths in finite samples. Scripta Mater. 56, 313-316.

Rao, S., Dimiduk, D., Parthasarathy, T., Uchic, M., Tang, M., Woodward, C., 2008. Athermal mechanisms of size-dependent crystal flow gleaned from three-dimensional discrete dislocation simulations. Acta Mater. 56, 3245-3259.

Rao, S.I., Dimiduk, D.M., Tang, M., Parthasarathy, T.A., Uchic, M.D., Woodward, C., 2007. Estimating the strength of single-ended dislocation sources in micron-sized single crystals. Philos. Mag. 87, 4777-4794.

Shan, Z.W., Mishra, R.K., Asif, S.A.S., Warren, O.L., Minor, A.M., 2008. Mechanical annealing and source-limited deformation in submicrometre-diameter Ni crystals. Nat. Mater. 7, 115119.

Sun, Q., Guo, Q., Yao, X., Xiao, L., Greer, J.R., Sun, J., 2011. Size effects in strength and plasticity of single-crystalline titanium micropillars with prismatic slip orientation. Scripta Mater. 65, 473-476.

Uchic, M.D., Dimiduk, D.M., Florando, J.N., Nix, W.D., 2004. Sample dimensions influence strength and crystal plasticity. Science 305, 986-989.

Volkert, C.A., Lilleodden, E.T., 2006. Size effects in the deformation of sub-micron Au columns. Philos. Mag. 86, 5567-5579.

Ye, J., Mishra, R.K., Sachdev, A.K., Minor, A.M., 2011. In situ TEM compression testing of Mg and Mg-0.2 wt.\% Ce single crystals. Scripta Mater. 64, 292-295. 
Zaiser, M., Bay, K., Hähner, P., 1999. Fractal analysis of deformation-induced dislocation patterns. Acta Mater. 47, 2463-2476.

Zaiser, M., Schwerdtfger, J., Schneider, A.S., Frick, C.P., Clark, B.G., Gruber, P.A., Arzt, E., 2008. Strain bursts in plastically deforming molybdenum micro- and nanopillars. Philosophical Magazine 88, 3861-3874.

Zuo, L., Ngan, A.H.W., 2006. Molecular dynamics study on compressive yield strength in $\mathrm{Ni}_{3} \mathrm{Al}$ micro-pillars. Philosophical Magazine Letters 86, 355-365. 


\section{Figure Captions}

Fig. 1. TEM montages and their digitized bitmaps of Al bulk specimens: (a) undeformed; (b) 7\% cold-rolled reduction; (c) 15\% cold-rolled reduction. Montage (a) was taken at $g=[\overline{1} \overline{1} 1]$ near the [101] pole, (b) and (c) were taken at $g=[\overline{1} \overline{1} 1]$ near the [112] pole. The side length for all micrographs is $9 \mu \mathrm{m}$.

Fig. 2. Determination of fractal dimensions of initial dislocation structures by (a) the boxcounting method, and (b) mass-dimension counting. The line segment in each plot shows the range used to obtain the approximate fractal dimension.

Fig. 3. Size dependence of yield strength of Al micro-pillars. The dashed lines indicate the yield strength of the bulk specimens.

Fig. 4. Experimental and theoretical power-law exponent in size dependence of strength versus the pre-strain of the initial dislocation pattern. Theoretical $m$ ' values for the $7 \%$ and $15 \%$ prestrained cases are calculated from the 2-D fractal model with eqn. (17) in Section 5, while that for the $0 \%$ prestrained case is calculated from the non-fractal model in the Appendix.

Fig. 5. Experimental and theoretical plots of survival probability versus applied stress for micropillars with (a) 7\% pre-straining and (b) 15\% pre-straining.

Fig. 6. (a) Non-power-law curve fitting of $N(\Delta x)$. (b) Probability density $p_{2-D}(L)$. (c) Numerically integrated $Y(\sigma)$ plotted in double logarithmic format. For $\sigma$ values from 20 to 170 MPa, $Y(\sigma)$ could be approximately fitted to a power-law equation $Y \propto \sigma^{3.20}$ as indicated by the red line.

Fig. 7. Experimental and theoretical plots of survival probability versus applied stress compression of micro-pillars without pre-straining. 

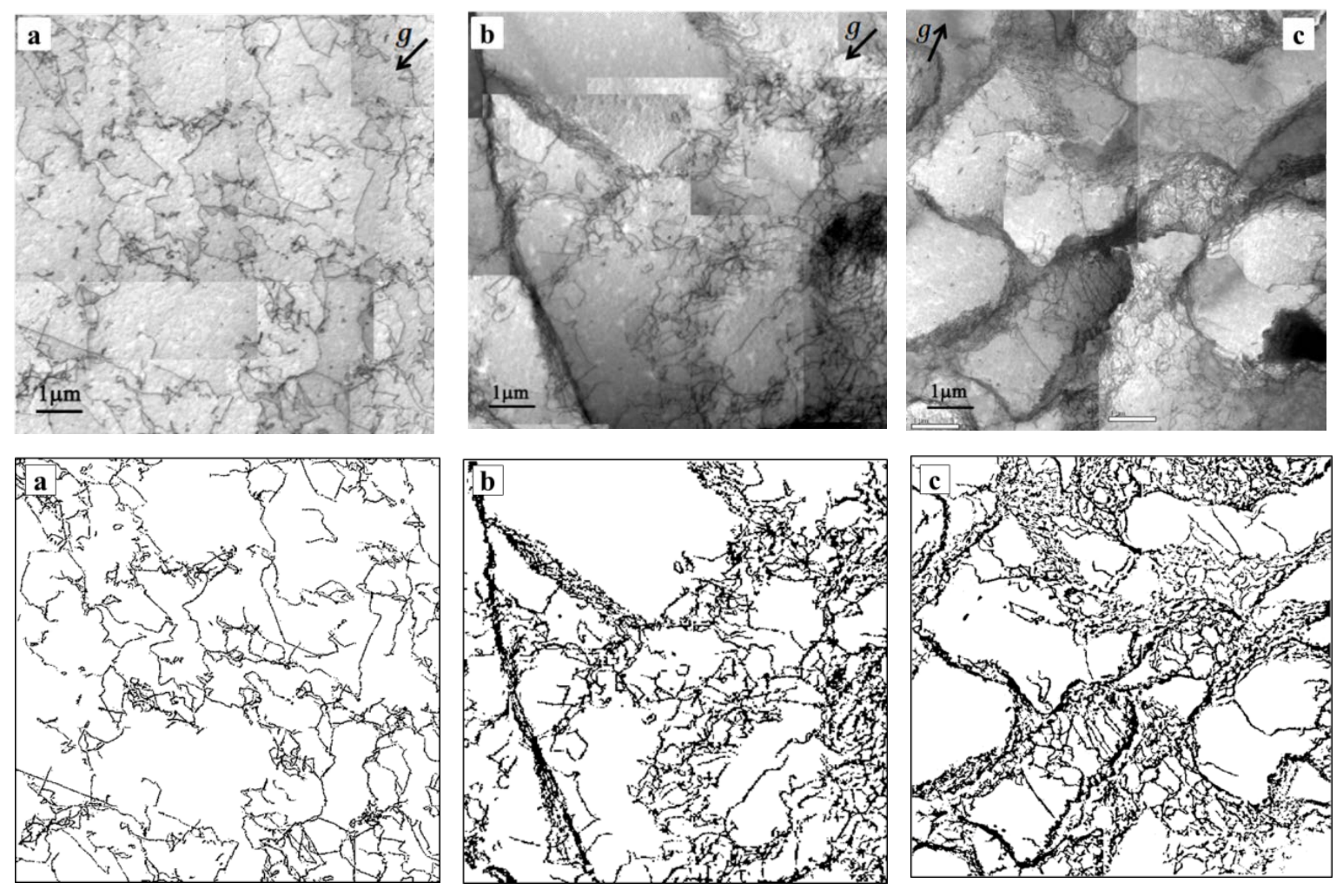

Fig. 1. TEM montages and their digitized bitmaps of Al bulk specimens: (a) undeformed; (b) 7\% cold-rolled reduction; (c) 15\% cold-rolled reduction. Montage (a) was taken at $g=[\overline{1} \overline{1} 1]$ near the [101] pole, (b) and (c) were taken at $g=[\overline{1} \overline{1} 1]$ near the [112] pole. The side length for all micrographs is $9 \mu \mathrm{m}$. 


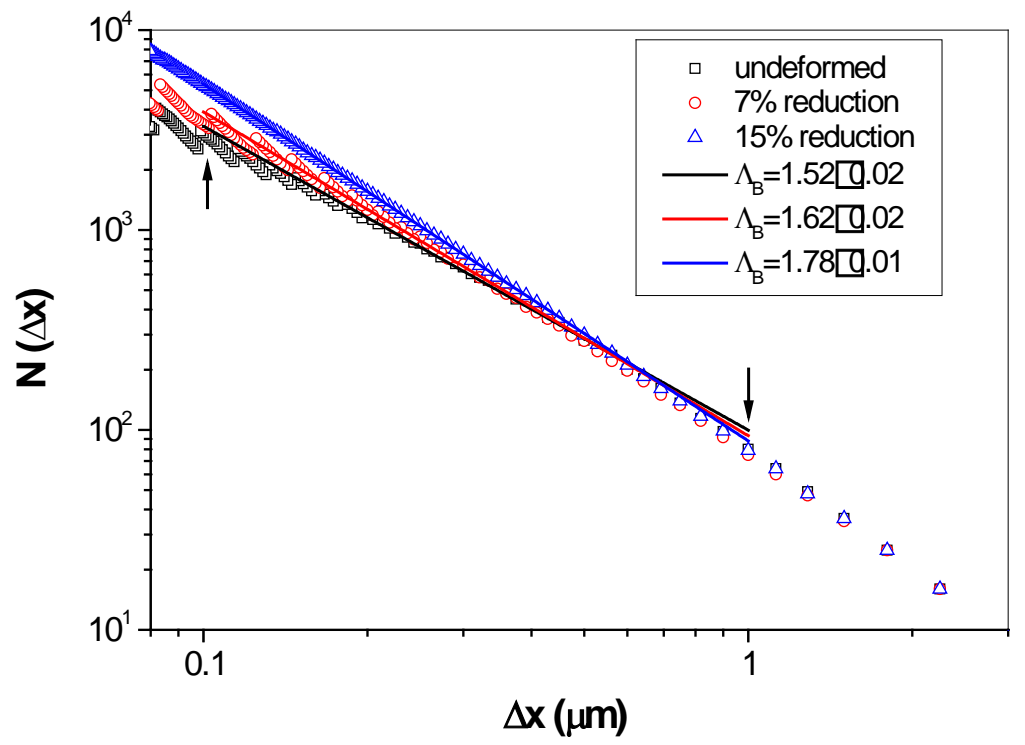

(a)

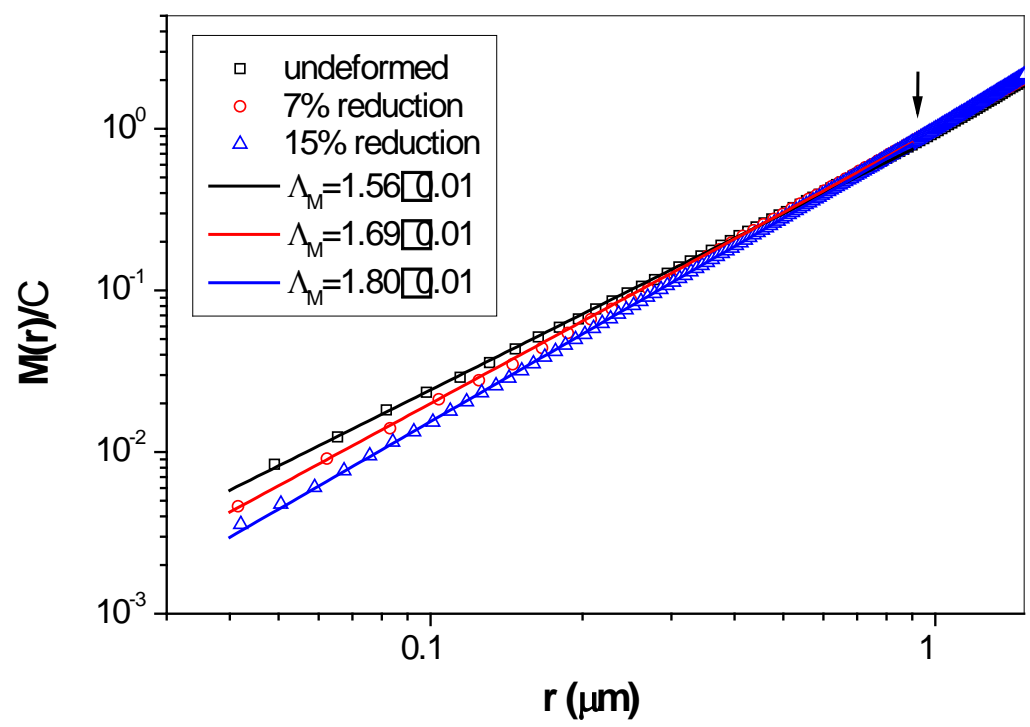

(b)

Fig. 2. Determination of fractal dimensions of initial dislocation structures by (a) the boxcounting method, and (b) mass-dimension counting. The line segment in each plot shows the range used to obtain the approximate fractal dimension. 


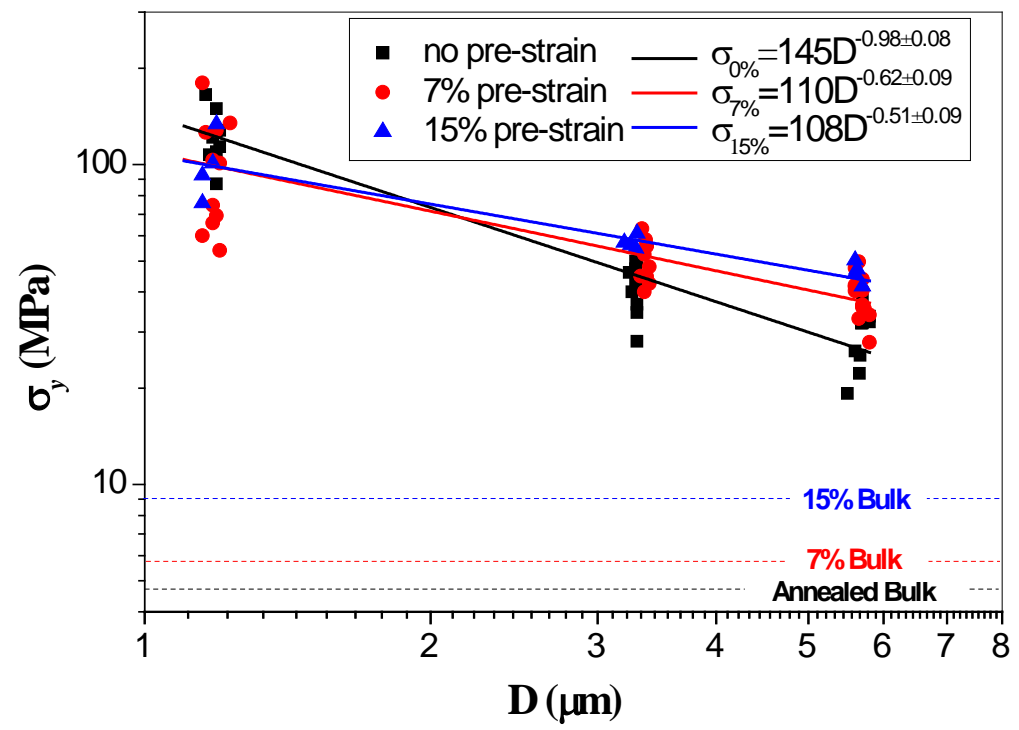

Fig. 3. Size dependence of yield strength of Al micro-pillars. The dashed lines indicate the yield strength of the bulk specimens.

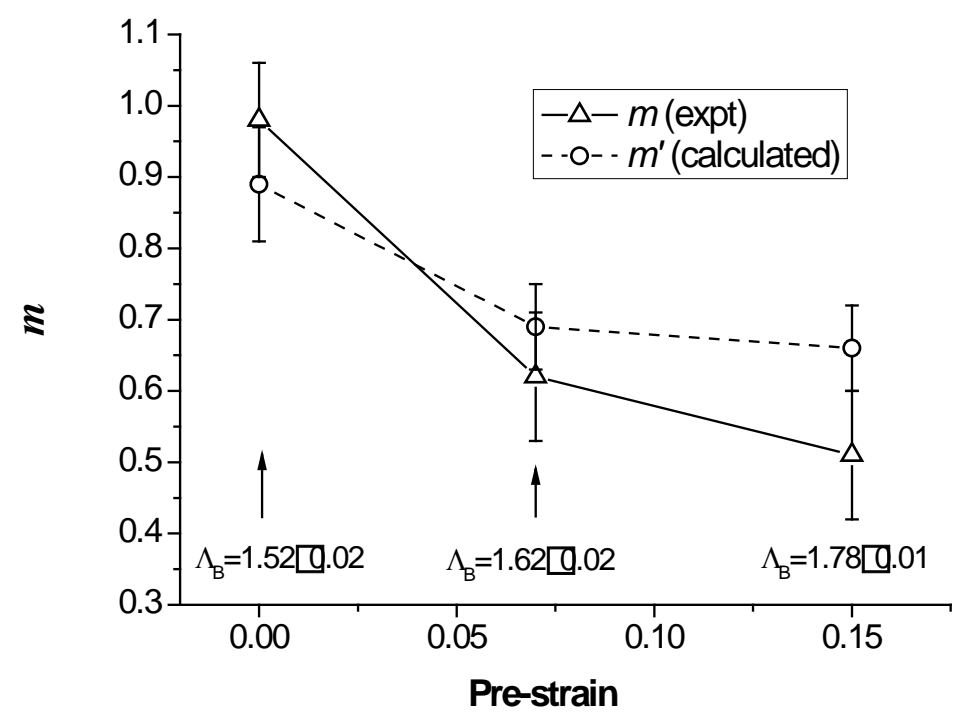

Fig. 4. Experimental and theoretical power-law exponent in size dependence of strength versus the pre-strain of the initial dislocation pattern. Theoretical $m$ ' values for the $7 \%$ and $15 \%$ prestrained cases are calculated from the 2-D fractal model with eqn. (17) in Section 5, while that for the $0 \%$ prestrained case is calculated from the non-fractal model in the Appendix. 


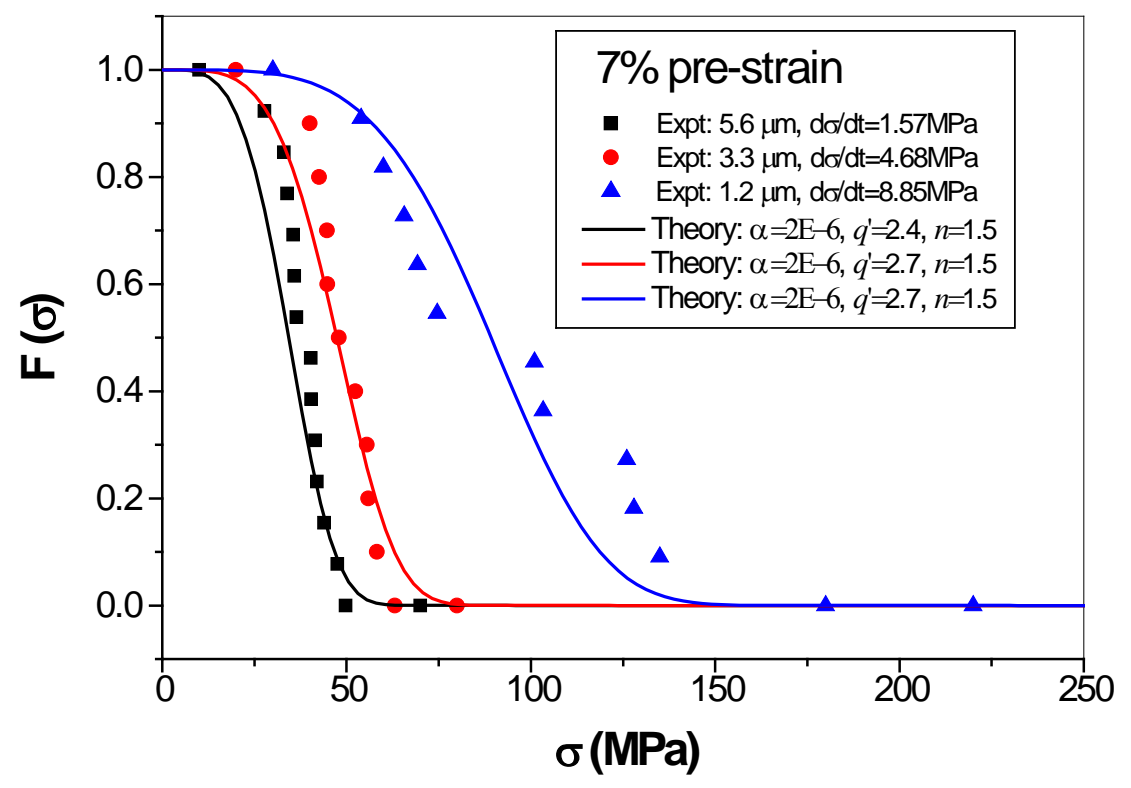

(a)

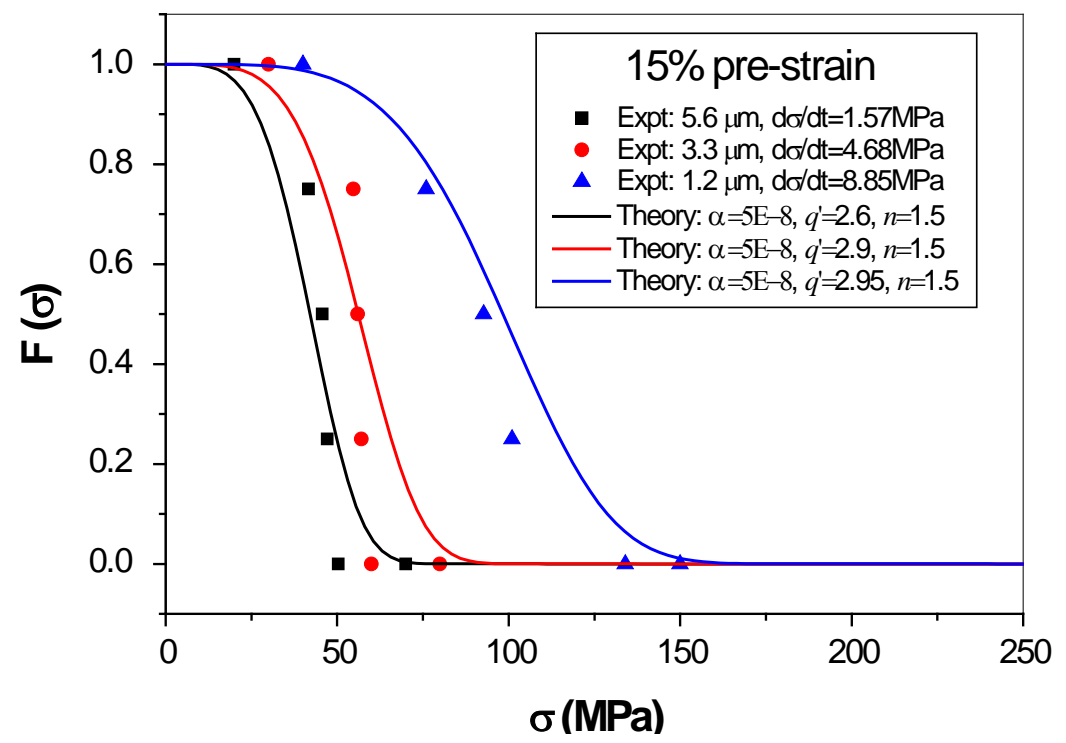

(b) 
Fig. 5. Experimental and theoretical plots of survival probability versus applied stress for micropillars with (a) 7\% pre-straining and (b) 15\% pre-straining.

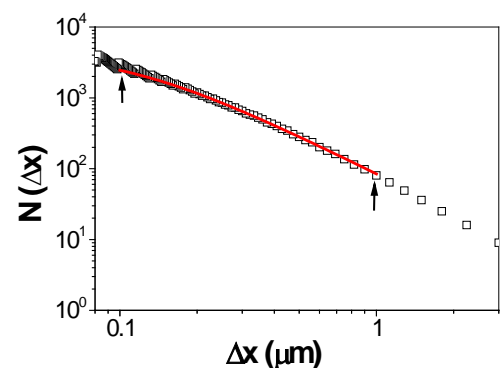

(a)

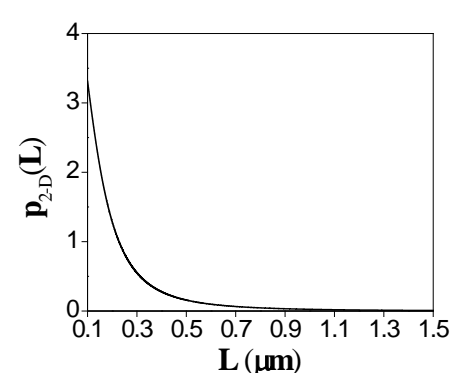

(b)

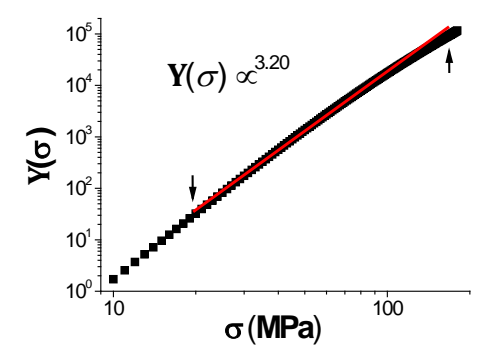

(c)

Fig. 6. (a) Non-power-law curve fitting of $N(\Delta x)$. (b) Probability density $p_{2-D}(L)$. (c) Numerically integrated $Y(\sigma)$ plotted in double logarithmic format. For $\sigma$ values from 20 to 170 MPa, $Y(\sigma)$ could be approximately fitted to a power-law equation $Y \propto \sigma^{3.20}$ as indicated by the red line.

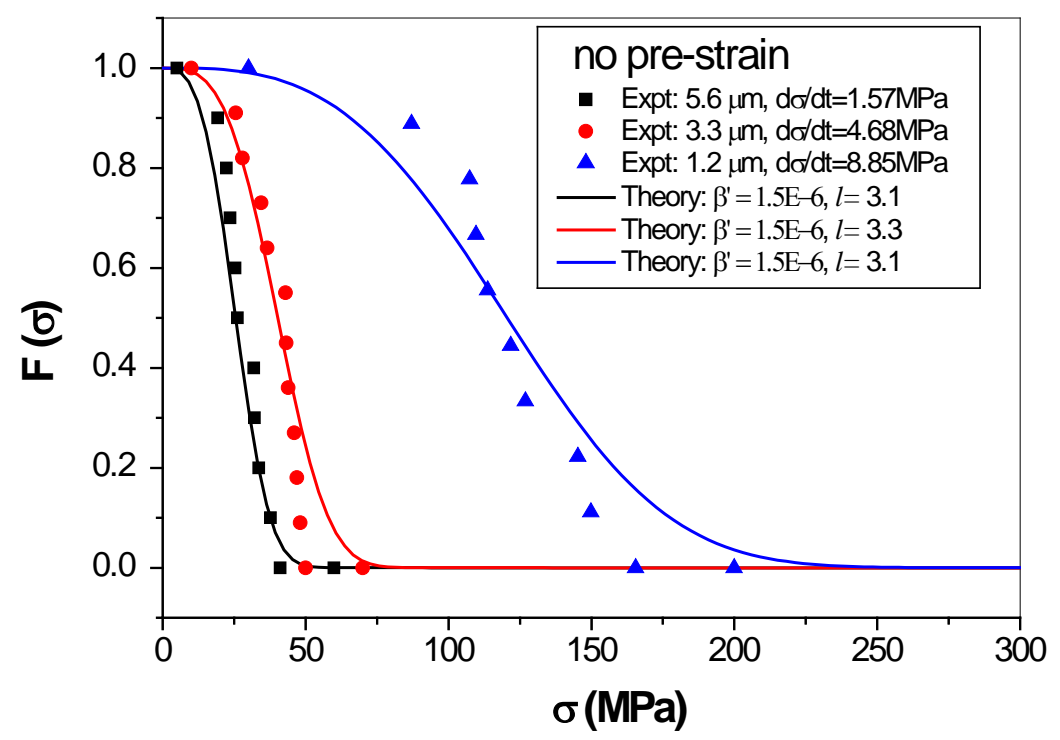

Fig. 7. Experimental and theoretical plots of survival probability versus applied stress compression of micro-pillars without pre-straining. 